\title{
EDITORIAL
}

\section{Investigación en pregrado, semilleros de investigación y creación del SemiLAC}

\author{
Alfonso J. Rodríguez-Morales ${ }^{1}$, Andrés Felipe López² \\ ${ }^{1}$ Docente, codirector del Grupo de Investigación Salud Pública e Infección, Facultad de Ciencias de \\ la Salud, Universidad Tecnológica de Pereira, Pereira, Colombia. Miembro de la World Association of \\ Medical Editors y del Council of Science Editors \\ ${ }^{2}$ Estudiante de Medicina, miembro del Semillero de Investigación Salud Pública e Infección, Facultad \\ de Ciencias de la Salud, Universidad Tecnológica de Pereira, Pereira, Colombia
}

Correspondencia: arodriguezm@utp.edu.co

La utopía es, según Eduardo Galeano, el horizonte hacia el cual avanzan nuestros sueños; si caminamos diez pasos, el horizonte se mueve diez pasos hacia adelante, y esta es la gran razón de la utopía, hacernos avanzar, hacernos perseguir aquello que parece inalcanzable. De igual manera, la investigación científica en salud debe encaminarse hacia grandes metas, reales y actualizadas con el estado de salud de la población investigada; si apuntamos nuestros esfuerzos hacia estos grandes sueños, haremos pequeños avances bien encaminados.

Los semilleros de investigación se vuelven una fuente de inspiración para los estudiantes de pregrado, especialmente en salud y en medicina. Según nuestra experiencia, son una alternativa a las investigaciones realizadas dentro del plan de estudio como parte de un sistema de calificación obligatorio. Cuando un estudiante se vincula a un semillero, lo hace a partir de la voluntad, y esto debe ser una premisa en la investigación científica, puesto que buscan responder el qué, el cómo y el porqué de aquello que les causa curiosidad. Investigar debe ser un acto de voluntad, sin perder de vista la priorización, pero definitivamente, fomentándolo e incentivándolo institucional y académicamente. 
A partir del año 2004, se inició en Colombia un proyecto para identificar prioridades de investigación en salud, utilizando el método de la matriz combinada del Global Forum for Health Research. En este, se pusieron en práctica dos trayectos metodológicos principales: por una parte, el diseño y aplicación de un método para ponderar u ordenar, de manera cualitativa y cuantitativa, los problemas de investigación en salud; por otra parte, la construcción de consensos con investigadores y representantes de comunidades científicas, encontrándose que los problemas predominantes de salud en Colombia son las enfermedades crónicas, las enfermedades infecciosas emergentes, la tuberculosis, la lepra o enfermedad de Hansen, las infecciones asociadas a la atención en salud (anteriormente llamadas hospitalarias), e infecciones de transmisión sexual y VIH/SIDA (1). De manera más específica, las lesiones por causa violenta, la enfermedad respiratoria aguda y crónica, los problemas perinatales, los problemas nutricionales y las enfermedades congénitas, causan $56 \%$ de la carga de enfermedad en el país, y se destina tan solo el $10 \%$ de los recursos para esto, en contraste con el $25 \%$ destinado a las enfermedades infecciosas (2).

En esas necesidades de investigación existe una clara oportunidad, no solo de hacer aportes a problemas que requieren soluciones desde la investigación, sino de fomentar una activa participación de los estudiantes de pregrado, que además, podrán adquirir tempranamente herramientas que requerirán posteriormente en su futuro profesional. Más aún, la curva de aprendizaje en investigación se iniciará tempranamente, contando entonces con una experiencia más amplia al graduarse como profesionales.

Los semilleros, como su nombre lo indican, son un espacio para dar origen y principio, de que nazcan y se propaguen los futuros investigadores. Como tal, debe entenderse la importancia de esta actividad e, incluso, debe reconocerse a nivel no solo institucional, dentro de las universidades, sino que debe tenerse un mayor registro de ello. Es por eso que, si desde el Departamento Administrativo de Ciencia, Tecnología e Innovación de Colombia, Colciencias, existe para las instituciones el InstituLAC, registro de la hoja de vida de actividades y producción científica de las instituciones, con su correspondiente GrupLAC para los grupos de investigación y CVLAC para los investigadores, debería existir también el SemiLAC. EI SemiLAC, así como el GrupLAC, debería contar con el registro de actividades y producción científica de los semilleros de investigación y sus estudiantes miembros, que ya cuentan a su vez con el llamado CvLAC. 
Finalmente, es siempre importante recordar que, al fomentar y enseñar a investigar en pregrado, especialmente mediante los semilleros de investigación, es fundamental instruir sobre la importancia de concluir debidamente las investigaciones, proceso que no termina hasta que se publican. Investigar y publicar es un binomio indivisible que debe iniciarse en pregrado $(3,4)$. De esta forma, podremos crear una cultura de investigación y publicación que tenderá, no sólo a cerrar la brecha de investigación que existe con grandes instituciones y países desarrollados, sino también, a fomentar la solución de problemas y generar desarrollo sostenible y búsqueda de mejores condiciones de salud, basados en soluciones propias, para nuestros pueblos en Colombia y en América Latina.

\section{Conflicto de intereses}

Los autores no tienen conflicto de intereses.

\section{Referencias}

1. Agudelo CA, de la Hoz F, Mojica MJ, Eslava JC, Robledo R, Cifuentes P, et al. Prioridades de investigación en salud en Colombia: perspectiva de los investigadores. Rev Salud Pública. (Bogotá) 2009;11:301-9.

2. Global Forum for Health Research. The 10/90 Report on Health Research 2001-2002. Geneva: Global forum for Health Research; 2002.

3. Huamaní C, Mayta-Tristán P, Rodríguez-Morales AJ. Publicar desde pregrado. Interciencia. 2008;33:785.

4. Pereyra-Elías P, Rodríguez-Morales AJ, Mayta-Tristán P. Undergraduate publication in Latin America: Role of medical students' scientific societies. Med Teach. 2011;33:594. 